Abstract

\title{
Selenocystine Modified Screen-Printed Carbon Electrode as an Alternative Sensor for the Voltammetric Determination of Metal Ions ${ }^{\dagger}$
}

\author{
Núria Serrano *, Jaume Puy-Llovera, Clara Pérez-Ràfols, José Manuel Díaz-Cruz, Cristina Ariño \\ and Miquel Esteban \\ 1 Department of Chemical Engineering and Analytical Chemistry, Faculty of Chemistry, University of \\ Barcelona, 08028 Barcelona, Spain; jaume.pllov7@gmail.com (J.P.-L.); claraperezrafols@ub.edu (C.P.-R.); \\ josemanuel.diaz@ub.edu (J.M.D.-C.); cristina.arino@ub.edu (C.A.); miquelestebanc@ub.edu (M.E.) \\ * Correspondence: nuria.serrano@ub.edu \\ + Presented at the 5th International Symposium on Sensor Science (I3S 2017), Barcelona, Spain, \\ 27-29 September 2017.
}

Published: 29 November 2017

Heavy metals are present in the environment from both natural and anthropogenic sources. Their bioaccumulation and persistence constitutes a serious threat to human health, demanding suitable methods for heavy metal monitoring. In this sense, anodic stripping voltammetry is especially appropriate for measuring trace metals. Conventionally, stripping techniques were connected to the use of mercury-based electrodes. Nevertheless, concerns about mercury toxicity have led to focus on the development of more environmentally friendly alternatives such as chemically modified electrodes (CME). The commercial availability of screen-printed electrodes (SPE) has largely expanded the field of application of modified electrodes as substitutes for mercury.

The molecule immobilization procedure is a key aspect in the design of CMEs. Particularly, the molecule immobilization based on aryl diazonium salt anchored on the electrode surface has proven to be a suitable approach for the development of sensors.

This work focuses on the development of a selenocystine modified sensor via electrografting on a screen-printed carbon-nanofiber substrate (SeCyst-SPCNFE). This sensor was applied to the determination of $\mathrm{Cd}(\mathrm{II})$ and $\mathrm{Pb}$ (II) and compared to a sensor modified with L-Cystine (CystSPCNFE) providing a better analytical performance. Finally, the applicability of SeCyst-SPCNFE was tested in a wastewater sample.

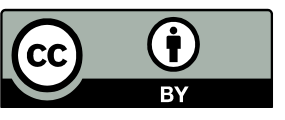

(C) 2017 by the authors. Licensee MDPI, Basel, Switzerland. This article is an open access article distributed under the terms and conditions of the Creative Commons Attribution (CC BY) license (http://creativecommons.org/licenses/by/4.0/). 